УДК 513.88

Mean Value Theorem for Harmonic Functions on Cayley Tree

Farrukh T. Ishankulov*

Samarkand State University

Universitetsky bulvar, 15

140104,Samarkand

Uzbekistan

Received 06.09.2014, received in revised form 06.10.2014, accepted 24.11.2014

An analog of the mean value theorem for harmonic functions on Cayley tree is proved in this paper.

Keywords: harmonic function, Caylee tree, mean value.

\title{
Introduction
}

A tree is connected acyclic graph. One special case of such graph is a Cayley tree. A Cayley tree is an infinite tree in which each vertex has exactly $k+1$ incident edges (the Cayley tree of order $k \geqslant 1)$. Let $\Gamma^{k}=(V, L)$ be the Cayley tree of order $k \geqslant 1$, where $V$ and $L$ are the vertex set and the edge set, respectively.

If $x, y \in V$ are the endpoints of an edge $l \in L$ then $x$ and $y$ are said to be adjacent. In this case we write $l=\langle x, y\rangle$. The distance on the Cayley tree $d(x, y), x, y \in V$ is determined by the formula

$$
\begin{array}{r}
d(x, y)=\min \left\{d: \exists x=x_{0}, x_{1}, \ldots, x_{d-1}, x_{d}=y \in V\right. \text { where } \\
\left.<x_{0}, x_{1}>, \ldots,<x_{d-1}, x_{d}>\text { are adjacent }\right\} .
\end{array}
$$

The sequence $\pi=\left\{x=x_{0}, x_{1}, \ldots, x_{d-1}, x_{d}=y \in V\right\}$ is referred to as path from $x$ to $y$.

Definition 1. A function $u: V \rightarrow R$ is said to be harmonic on Cayley tree if it satisfies the discrete Laplace equation

$$
u(x)=\frac{1}{k+1} \sum_{y \in W_{1}(x)} u(y),
$$

where $W_{1}(x)$ is the set of vertices adjacent to $x$.

To define a discrete ball and sphere [1] let us fix a point $x \in V$.

Definition 2. The set

$$
B_{n}=\{y \in V: d(x, y) \leqslant n\}
$$

is said to be a ball of radius $n$ and center $x$. The set $W_{n}=\{y \in V: d(x, y)=n\}$ is a discrete sphere of radius $n$ and center $x \in V$.

The mean value theorem plays an important role in the classical potential theory. According to this theorem, the value of harmonic function at the center of a sphere is equal to arithmetic average of its values on the surface of this sphere. This theorem can be also formulated for a ball: the value of harmonic function at the center of the ball is equal to arithmetic average of its values in points of this ball.

The aim of this paper is to proof an analog of the mean value theorem for harmonic functions on Cayley tree.

*fandor83@mail.ru

(c) Siberian Federal University. All rights reserved 


\section{Mean value theorems}

Theorem 1. If $u(x)$ is a harmonic function on $\Gamma^{k}=(V, L)$ then for any vertex $x \in V$ and for any natural number $m$ the following relation holds

$$
u(x)=\frac{1}{\left|W_{m}(x)\right|} \sum_{y \in W_{m}(x)} u(y),
$$

where $\left|W_{m}(x)\right|=k^{m-1}(k+1)$ is the number of points of sphere $W_{m}(x)$.

Proof. We prove by induction. For $m=1$ relation (2) has the same from as (1). For $m=2$ relation (2) has form

$$
u(x)=\frac{1}{k(k+1)} \sum_{y \in W_{2}(x)} u(y) .
$$

Let us prove relation (3). By using relation (1), we have

$$
\begin{aligned}
u(x)=\frac{1}{k+1} \sum_{y \in W_{1}(x)} u(y)= & \frac{1}{k+1} \sum_{y \in W_{1}(x)}\left(\frac{1}{k+1} \sum_{z \in W_{1}(x)} u(z)\right)=\frac{1}{(k+1)^{2}} \sum_{y \in W_{1}(x)} \sum_{z \in W_{1}(y)} u(x)= \\
& =\frac{1}{(k+1)^{2}}\left[(k+1) u(x)+\sum_{y \in W_{2}(x)} u(y)\right] .
\end{aligned}
$$

From the last relation we find $u(x)$ :

$$
u(x)=\frac{1}{k(k+1)} \sum_{y \in W_{2}(x)} u(y) .
$$

Suppose that relation (2) is true for $m=\overline{1, n}$ :

$$
u(x)=\frac{1}{k^{n-1}(k+1)} \sum_{y \in W_{n}(x)} u(y) .
$$

Using (4), we prove relation (2) for $m=n+1$ :

$$
\begin{aligned}
u(x) & =\frac{1}{k^{n-1}(k+1)} \sum_{y \in W_{n}(x)} u(y)=\frac{1}{k^{n-1}(k+1)} \sum_{y \in W_{n}(x)}\left(\frac{1}{k+1} \sum_{z \in W_{1}(y)} u(z)\right)= \\
& =\frac{1}{k^{n-1}(k+1)^{2}} \sum_{y \in W_{n}(x)} \sum_{z \in W_{1}(z)} u(z)=\frac{1}{k^{n-1}(k+1)^{2}}\left(k \sum_{t \in W_{n-1}} u(t)+\sum_{z \in W_{n+1}} u(z)\right) .
\end{aligned}
$$

By induction we have $\sum_{t \in W_{n-1}} u(t)=\left(k^{n-2}(k+1)\right) u(x)$. Hence, from (5) we obtain $u(x)=\frac{1}{k^{n-1}(k+1)^{2}}\left(k\left(k^{n-2}(k+1)\right) u(x)+\sum_{z \in W_{n+1}} u(z)\right)=\frac{1}{k+1} u(x)+\frac{1}{k^{n-1}(k+1)^{2}} \sum_{z \in W_{n+1}} u(z)$.

Hence we have

$$
u(x)=\frac{1}{k^{n}(k+1)} \sum_{y \in W_{n+1}(x)} u(y)
$$

The following theorem is a discrete analog of the mean value theorem for a ball. 
Theorem 2. If $u(x)$ is a harmonic function on $\Gamma^{k}=(V, L)$ then for any vertex $x \in V$ and for any natural number $m$ the following relation holds

$$
u(x)=\frac{1}{\left|B_{n}(x)\right|} \sum_{y \in B_{n}(x)} u(y) .
$$

Proof. By theorem 1 for $\forall n \in N$ we have

$$
\sum_{i=0}^{n}\left|W_{i}(x)\right| u(x)=\sum_{i=0}^{n} \sum_{y \in W_{i}(x)} u(y) .
$$

Note that $\sum_{i=0}^{n}\left|W_{i}(x)\right|=\left|B_{n}(x)\right|=\frac{k^{n+1}+k^{n}-2}{k-1}$ and from (7) we obtain

$$
u(x)=\frac{1}{\left|B_{n}(x)\right|} \sum_{y \in B_{n}(x)} u(y) .
$$

\section{References}

[1] A.Grigoryan, Analysis on Graphs, Lecture Notes, University of Bielefeld, WS 2011/12.

[2] S.G.Mikhlin, Linear partial differential equations, Moskow, Vysshaya Shkola, 1977 (in Russian).

[3] P.M.Soardi, Potential theory of infinite network, V. 1590, Lecture Notes in Math. SpringerVerlag, Berlin, 1994.

[4] U.A.Rozikov, F.T.Ishankulov, Description of periodic p-harmonic functions on Cayley tree, Nonlinear Differ. Equ. Appl., 17(2010), 15-160.

\section{Теорема о среднем значении для гармонических функций на деревьях Кэли}

\section{Фаррух Т. Ишанкулов}

Доказана теорема о среднем значении для гармонических функций на деревъях Кэли.

Ключевые слова: гармоническая функиия, дерево Кэли, среднее значение. 